\title{
Double-barrel Damus-Kaye-Stansel operation is better than end-to-side Damus-Kaye-Stansel operation for preserving the pulmonary valve function: The importance of preserving the shape of the pulmonary sinus
}

\author{
Yasuhiro Fujii, $\mathrm{PhD},{ }^{\text {a }}$ Shingo Kasahara, $\mathrm{PhD},{ }^{\mathrm{a}}$ Yasuhiro Kotani, $\mathrm{PhD},{ }^{\mathrm{a}}$ Masami Takagaki, $\mathrm{PhD},{ }^{\mathrm{a}}$ \\ Sadahiko Arai, $\mathrm{PhD},{ }^{\mathrm{a}}$ Shin-ichi Otsuki, $\mathrm{PhD},{ }^{\mathrm{b}}$ and Shunji Sano, $\mathrm{PhD}^{\mathrm{a}}$
}

\begin{abstract}
Objective: The Damus-Kaye-Stansel operation sometimes results in deteriorating semilunar valve insufficiency. We verified the semilunar valve function after the Damus-Kaye-Stansel operation and compared the end-to-side Damus-Kaye-Stansel with the double-barrel Damus-Kaye-Stansel.
\end{abstract}

\begin{abstract}
Methods: Forty-seven patients who underwent the Damus-Kaye-Stansel operation between June 1993 and August 2008 were retrospectively reviewed. Any patient who underwent a Norwood-type operation was excluded. The median age at operation was 19 months (range, 0-276 months). Forty-five patients were Fontan candidates. Thirty-nine patients underwent pulmonary artery banding before the Damus-Kaye-Stansel operation. Twenty-two patients had undergone an arch repair previously. The semilunar valve function was evaluated by echocardiography.
\end{abstract}

\begin{abstract}
Results: Thirteen patients underwent the end-to-side Damus-Kaye-Stansel operation, and 34 patients underwent the double-barrel Damus-Kaye-Stansel operation. The mean follow-up period was $71 \pm 50$ months (range, $1-188$ months). Although there were 4 deaths, no death was related to the Damus-Kaye-Stansel procedure. Two of the patients with early death could not undergo a postoperative evaluation of the semilunar valves. The semilunar valve regurgitation mildly deteriorated in 7 patients (pulmonary regurgitation in 5 patients and aortic regurgitation in 2 patients). Pulmonary regurgitation deteriorated from none to mild in 1 patient, none to trivial in 2 patients, and trivial to mild in 2 patients. Both deteriorations in aortic regurgitation ranged from none to trivial. Semilunar valve regurgitation did not affect patients' circulatory condition. The end-to-side Damus-Kaye-Stansel operation more frequently caused a deterioration in pulmonary regurgitation than the double-barrel Damus-Kaye-Stansel operation $(4 / 11$ vs $1 / 34, P=.001)$. No surgical intervention for a systemic ventricular outflow obstruction was observed in the follow-up period.
\end{abstract}

Conclusions: The double-barrel Damus-Kaye-Stansel operation was found to be superior to the end-to-side Damus-Kaye-Stansel operation for the prevention of postoperative pulmonary regurgitation. (J Thorac Cardiovasc Surg 2011;141:193-9)

The Damus-Kaye-Stansel (DKS) operation contributes to an improved clinical outcome of the Fontan operation in patients with systemic ventricular outflow obstruction (SVOO). The DKS operation is aggressively performed for Fontan candidates who have an SVOO or the anatomic potential for SVOO, such as a double-inlet ventricle with transposition of the great arteries and tricuspid atresia with transposition of the great arteries.

\footnotetext{
From the Department of Cardiovascular Surgery ${ }^{\mathrm{a}}$ and Department of Pediatrics, ${ }^{\mathrm{b}}$ Okayama University Hospital, Okayama, Japan.

Disclosures: Authors have nothing to disclose with regard to commercial support.

Received for publication Dec 10, 2009; revisions received May 21, 2010; accepted for publication June 6, 2010; available ahead of print July 16, 2010.

Address for reprints: Yasuhiro Fujii, PhD, Department of Cardiovascular Surgery, Okayama University Hospital, 2-5-1 Shikata-cho, Kita-ku, Okayama-city, Okayama, 700-8558, Japan (E-mail: GZE00572@nifty.ne.jp). $0022-5223 / \$ 36.00$

Copyright (C) 2011 by The American Association for Thoracic Surgery doi:10.1016/j.jtcvs.2010.06.007
}

Damus, ${ }^{1}$ Kaye, ${ }^{2}$ and Stansel ${ }^{3}$ originally described an endto-side anastomosis between the main pulmonary artery and the ascending aorta (end-to-side DKS) to achieve a biventricular repair in patients with dextro-transposition of the great arteries. Waldman and colleagues ${ }^{4}$ reported a technical modification of the DKS operation in which the ascending aorta was sutured to a new bivalve single aorta, known as the "double-barrel method" (double-barrel DKS). The double-barrel DKS is the first choice for the DKS operation in the Okayama University Hospital because it is simpler and technically easier than the end-to-side DKS and no patch material is needed. The influence of the DKS operation on the semilunar valves, especially for the pulmonary valve, remains controversial. Past studies have observed mild or moderate postoperative pulmonary regurgitation (PR) in patients who underwent end-to-side DKS..$^{5-7}$ However, there have been few reports describing the pulmonary valve function after double-barrel DKS and which DKS operation is superior for pulmonary valve function has not been 


\section{Abbreviations and Acronyms \\ $\mathrm{AR}=$ aortic regurgitation \\ DKS = Damus-Kaye-Stansel \\ $\mathrm{PAB}=$ pulmonary artery banding \\ $\mathrm{PR}=$ pulmonary regurgitation \\ SVOO $=$ systemic ventricular outflow obstruction \\ $\mathrm{TCPC}=$ total cavopulmonary connection}

elucidated. The purpose of this study was to investigate the mid- to long-term outcome of patients who underwent the DKS operation and describe the effect on the semilunar valve function.

\section{PATIENTS AND METHODS \\ Study Subjects}

The medical and surgical records of patients with congenital heart disease who underwent the DKS operation in Okayama University Hospital between June 1993 and August 2008 were retrospectively reviewed. Any patients who underwent a Norwood-type operation were excluded.

\section{Data Collection and Measurements}

This study was approved by the institutional research ethics board at the Okayama University Hospital, and patient consent was waived. The data collected from medical record review included patient demographics, cardiac diagnosis, clinical condition, previous procedures, operative information, and postoperative and follow-up clinical status, including catheter and echocardiographic data. A significant SVOO was determined to be a systemic ventricular outflow tract Doppler flow velocity of greater than $2.0 \mathrm{~ms}$ of systemic ventricular outflow as confirmed by preoperative echocardiography or more than $5 \mathrm{~mm} \mathrm{Hg}$ of pressure gradient between the systemic ventricle and the ascending aorta verified by preoperative catheter examination. The semilunar valve function was evaluated using echocardiography by independent cardiac pediatricians who were all trained at Okayama University Hospital. However, the preoperative and postoperative pulmonary valve functions were not always evaluated by a pediatrician. The semilunar valve regurgitation was graded as none, trivial, mild, moderate, or severe. Thereafter, a "deterioration of the semilunar valve function" was diagnosed when the postoperative semilunar valve regurgitation was worse than the preoperative one.

\section{Selection of the Method of Damus-Kaye-Stansel Operation}

The double-barrel and end-to-side DKS operations were performed equally until 2001; however, the double-barrel DKS has been the first choice of treatment since 2002. The end-to-side DKS has been performed only in patients with an ascending aorta of insufficient diameter for the doublebarrel DKS. An almost equal diameter of the ascending aorta to the that of the pulmonary trunk was considered to be a good indication for the double-barrel DKS. All DKS operations were performed by only 1 surgeon.

\section{Surgical Procedures}

The DKS operation was performed using a conventional continuous flow cardiopulmonary bypass with mild to moderate hypothermia $\left(24^{\circ} \mathrm{C}-34^{\circ} \mathrm{C}\right)$ or circulatory arrest under deep hypothermia. The DKS anastomosis was created using polypropylene running sutures. The DKS operation without any patch material was the first choice. Figure 1 shows the types of DKS operation that were used. All of the semilunar valves were examined at the time of operation.

\section{Data Analysis}

To determine the effect of the DKS operation on the semilunar valve, these patients were divided into 2 groups according to the type of the DKS operation (the end-to-side DKS group and the double-barrel DKS group), and these groups were then compared statistically. Binary data were analyzed using Pearson's chi-square test. The Student $t$ test or Mann-Whitney $U$ test was used for comparisons of continuous data as appropriate. A multiple logistic regression analysis was used to compare the postoperative incidence of deterioration in PR. Odds ratios and confidence intervals were calculated as appropriate. Data are expressed as the mean \pm standard deviation or median and range as appropriate.

\section{RESULTS}

\section{Patient Characteristics}

Forty-seven patients with complex congenital heart disease underwent the DKS operation during the study period. The preoperative cardiac anatomic characteristics of these patients are summarized in Table 1. Forty-five patients were candidates for a Fontan operation, and 2 patients were candidates for a biventricular repair. The median age at the time of the operation was 19 months (range, 0-276 months), and the median body weight was $8.5 \mathrm{~kg}$ (range, $2.6-45 \mathrm{~kg}$ ). Thirty-three patients had a bulboventricular foramen in the systemic ventricular outflow tract. Twenty-two patients had arch anomalies, which included coarctation of the aorta in 15 patients and interruption of the aortic arch in 7 patients. Thirty-nine patients had undergone pulmonary artery banding (PAB) previously, and the median duration from the PAB to the DKS operation was 18 months (range, 3-108 months). Twenty-nine patients had a significant SVOO. The average preoperative blood flow velocity across the systemic ventricular outflow tract was $1.91 \pm 0.86 \mathrm{~ms}$ (range, 0.80-3.54 ms) with echocardiography. There was no preoperative instance of PR in 17 patients, whereas it was trivial in 29 patients and mild in 1 patient. Preoperative aortic regurgitation (AR) was not seen in 31 patients, was trivial in 15 patients, and was mild in 1 patient, as determined by echocardiography.

\section{Surgical Data}

The DKS operation was performed during a bidirectional Glenn procedure in 34 patients, before a bidirectional Glenn procedure in 4 patients, during a total cavopulmonary connection (TCPC) in 6 patients, during a TCPC takedown in 1 patient, and during a biventricular repair (repair of interruption of the aortic arch, closure of ventricular septal defect, and right ventricular outflow tract reconstruction) in 2 patients. Thirteen of the 47 patients underwent an end-to-side DKS, and an expanded polytetrafluoroethylene patch augmentation was used in 1 patient to prevent kinking of the anastomosis. Thirty-four patients underwent a doublebarrel DKS with no prosthetic materials. Five of the 47 patients underwent circulatory arrest with deep hypothermia. The other associated procedures were the enlargement of an atrial septal defect in 12 patients, reconstruction of the 
Double Barrel DKS

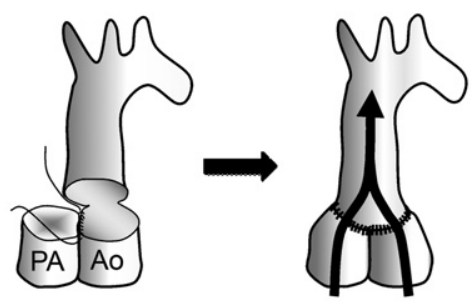

\section{End-to-Side DKS}

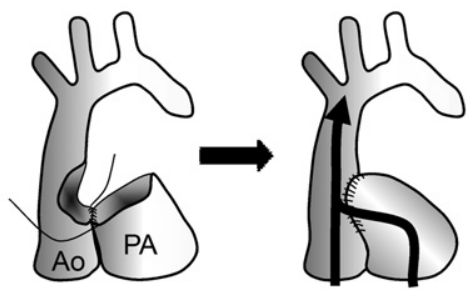

FIGURE 1. DKS types. When the double-barrel DKS is created, the posterior wall of the ascending aorta is uncut if possible to prevent bleeding after the anastomosis. The double-barrel DKS seems to have a smoother systemic ventricular outflow tract than the end-to-side DKS. DKS, Damus-Kaye-Stansel; $P A$, pulmonary artery; Ao, aorta.

pulmonary artery in 2 patients, resection of subaortic stenosis in 2 patients, a modified Blalock-Taussig shunt in 4 patients, tricuspid valve repair in 2 patients, and exchange of a pacemaker generator in 1 patient. The mean aortic crossclamp time was $60 \pm 23$ minutes (range, 23-122 minutes), and the mean cardiopulmonary bypass time was $126 \pm 60$ minutes (range, 67-419 minutes). All of the semilunar valves were found to have a normal appearance.

\section{Postoperative Outcomes}

There were 2 early deaths. One of these patients was a 5month-old female with tricuspid atresia and transposition of the great arteries who had previously undergone PAB. She underwent a semi-emergency bidirectional Glenn operation and the DKS operation because of heart failure with acidosis, severe hypoxia, and subaortic stenosis $(2.0 \mathrm{~ms}$ by echocardiography). She had necrosis of the intestine and died 18 days after the operation. The other patient was a 52-monthold female with a double-inlet left ventricle (situs solitus, L-loop, L-transposition). Although she initially underwent a TCPC without a DKS operation, low-output syndrome occurred as the result of SVOO, which deteriorated after the TCPC. As a result, an emergency DKS operation and TCPC takedown were performed on the first postoperative day; however, extracorporeal membranous oxygenation support was required because hypoxia and low left ventricular function did not improve. The patient died of sepsis and multiorgan dysfunction. There were 2 late deaths, and the mean follow-up time of the 45 early survivors was $75 \pm$ 49 months (range, 6-188 months). Both of the patients with late death underwent the DKS operation with a bidirec-
TABLE 1. Summary of cardiac anatomic diagnoses

\begin{tabular}{lr}
\hline \multicolumn{1}{c}{ Functional single ventricle $(\mathbf{n}=\mathbf{4 5})$} & 15 \\
\hline SLV & 7 \\
SLV (SLL) & 5 \\
SLV (SDD) & 3 \\
Others & 3 \\
TA + TGA & 4 \\
SRV & 1 \\
SRV (SDL) & 2 \\
Others & 5 \\
MA or severe MS with hypoplastic LV & 1 \\
MA + DORV & 1 \\
MS + DORV & 1 \\
DORV & 1 \\
DORV (SDN) + straddling MV & 1 \\
DORV (SDN) + CAVC + Swiss cheese-like muscular VSD & 1 \\
DORV (SDN) + CAVC + hypoplastic LV & 1 \\
DORV (SDN) + inlet VSD & 1 \\
DORV (SDN) + multiple VSDs & 1 \\
DORV (SDD) + CAVC & 1 \\
DORV (SDD) + CCH & 1 \\
DORV (SDD) + straddling MV & 1 \\
DORV (SLD) + hypoplastic LV & 1 \\
DORV (SLD) + PS & 1 \\
DORV (SLD) + CCH + straddling of MV & 1 \\
DORV (IDL) + CAVC & 1 \\
TBH + straddling of MV & 1 \\
TGA & 1 \\
d-TGA + VSD + straddling of MV & 1 \\
d-TGA + Swiss cheese-like muscular VSD & 1 \\
l-TGA + VSD + PS & 1 \\
AS + MS + VSD & 1 \\
Two ventricular heart (n = 2) & 1 \\
IAA + VSD & 1 \\
IAA + DORV & 1 \\
\hline
\end{tabular}

$A V S D$, Atrioventricular septal defect; $A S$, aortic stenosis; $C C H$, crisscross heart $D O R V$, double outlet right ventricle; $I A A$, interruption of the aortic $\operatorname{arch} I D L$, situs inversus, D-loop, L-transposition; $L V$, left ventricle; $M A$, mitral atresia; $M S$, mitral stenosis; $M V$, mitral valve; $P S$, pulmonary stenosis; $S D D$, situs solitus, D-loop, D-transposition; $S D L$, situs solitus, D-loop, L-transposition; $S D N$, situs solitus, D-loop, Normal position; $S L D$, situs solitus, L-loop, D-transposition; $S L L$, situs solitus, L-loop, L-transposition; $S L V$, single left ventricle; $S R V$, single right ventricle; $T A$, tricuspid atresia; $T B H$, Taussig-Bing heart; $T G A$, transposition of the great arteries; $V S D$, ventricular septal defect.

tional Glenn operation and a subsequent Fontan completion. However, they died of mediastinitis and pneumonia after the Fontan completion. There were no deaths related to the DKS operation. There were no re-do DKS operations or surgical interventions for SVOO. No obstruction of the left pulmonary artery caused by compression of the bulbous pulmonary trunk after DKS was observed. Forty-two of the 45 Fontan candidates underwent a Fontan completion. Postoperative PR was not observed in 13 patients, was trivial in 28 patients, and was mild in 4 patients. Postoperative AR was not seen in 27 patients, was trivial in 17 patients, and was mild in 1 patient. AR and PR were not evaluated postoperatively in the 2 patients who died early. A mild deterioration 
TABLE 2. Comparison of the two techniques for the Damus-Kaye-Stansel anastomosis

\begin{tabular}{|c|c|c|c|c|c|c|c|c|c|}
\hline & \multirow{2}{*}{\multicolumn{2}{|c|}{$\begin{array}{c}\text { End-to-side DKS } \\
\quad(\mathrm{n}=13)\end{array}$}} & \multirow{2}{*}{\multicolumn{2}{|c|}{$\begin{array}{c}\text { Double-barrel DKS } \\
(\mathbf{n}=\mathbf{3 4})\end{array}$}} & \multicolumn{2}{|c|}{ Univariate } & \multicolumn{3}{|c|}{ Multivariate } \\
\hline & & & & & $P$ & OR & $P$ & OR & CI \\
\hline Age at DKS (mo) & $12(0-112)$ & & $22(1-276)$ & & .644 & & & & \\
\hline Period & June 1993 to January 2008 & & March 1996 to August 2008 & & & & & & \\
\hline Previous PAB & $10 / 13$ & & $29 / 34$ & & .495 & & & & \\
\hline $\begin{array}{l}\text { Duration from } \\
\text { the previous PAB }(\mathrm{mo})\end{array}$ & $13(3-51)$ & & $21(3-108)$ & & .422 & & & & \\
\hline Significant SVOO & $10 / 13$ & & $19 / 34$ & & .184 & & & & \\
\hline Arch anomaly & $8 / 13(\mathrm{CoA} 5$, IAA 3$)$ & & $14 / 34(\mathrm{CoA} 10, \mathrm{IAA} 4)$ & & .211 & & & & \\
\hline \multirow[t]{3}{*}{ Preoperative AR } & none: & 9 & none: & 22 & .908 & & & & \\
\hline & trivial: & 3 & trivial: & 12 & & & & & \\
\hline & mild: & 1 & & & & & & & \\
\hline \multirow[t]{3}{*}{ Preoperative PR } & none: & 7 & none: & 10 & .110 & & & & \\
\hline & trivial: & 6 & trivial: & 23 & & & & & \\
\hline & & & mild: & 1 & & & & & \\
\hline Anomaly of PV & 0 & & 0 & & & & & & \\
\hline CPBT (min) & $144 \pm 93(75-419)$ & & $118 \pm 42(67-239)$ & & .348 & & & & \\
\hline ACCT (min) & $60 \pm 21(27-104)$ & & $60 \pm 25(23-122)$ & & .886 & & & & \\
\hline Follow-up (mo) & $75 \pm 56(0.6-188)$ & & $70 \pm 48(6-161)$ & & .740 & & & & \\
\hline \multirow[t]{3}{*}{ Postoperative AR } & none: & 7 & none: & 20 & .969 & & & & \\
\hline & trivial: & 3 & trivial: & 14 & & & & & \\
\hline & mild: & 1 & & & & & & & \\
\hline \multirow[t]{3}{*}{ Postoperative PR } & none: & 4 & none: & 9 & .687 & & & & \\
\hline & trivial: & 4 & trivial: & 24 & & & & & \\
\hline & mild: & 3 & mild: & 1 & & & & & \\
\hline \multirow[t]{2}{*}{ Deterioration of $\mathrm{AR}$} & $0 / 11$ & & $2 / 34$ & & .048 & & & & \\
\hline & & & none $\rightarrow$ trivial: & 2 & & & & & \\
\hline \multirow[t]{4}{*}{ Deterioration of PR } & $4 / 11$ & & $1 / 34$ & & .001 & 0.073 & .023 & 0.068 & $0.007-0.688$ \\
\hline & none $\rightarrow$ trivial: & 1 & none $\rightarrow$ trivial: & 1 & & & & & \\
\hline & none $\rightarrow$ mild: & 2 & & & & & & & \\
\hline & trivial $\rightarrow$ mild: & 1 & & & & & & & \\
\hline
\end{tabular}

$\overline{D K S}$, Damus-Kaye-Stansel; $A C C T$, aortic crossclamp time; $A R$, aortic regurgitation; $C I$, confidence interval; $C O A$, coarctation of the aorta; $C P B T$, cardiopulmonary bypass time, IAA; interruption of the aortic arch, $O R$, odds ratio; $P A B$, pulmonary artery banding; $P R$, pulmonary regurgitation; $P V$, pulmonary valve; $S V O O$, systemic ventricular outflow obstruction.

in $\mathrm{PR}$ was observed in 5 patients. Four of the 5 patients underwent $\mathrm{PAB}$ before the DKS operation. However, PAB was not found to be a significant risk factor for PR deterioration $(P=.799)$. A mild deterioration in AR was found in 2 patients.

\section{End-to-Side Damus-Kaye-Stansel Versus Double- Barrel Damus-Kaye-Stansel}

Table 2 shows a comparison of the characteristics between the patients who underwent an end-to-side DKS and the patients who underwent a double-barrel DKS. There was no significant difference in age at the DKS operation, the number of patients who had undergone a PAB before the DKS operation, the duration from the PAB to the DKS operation, the number of patients who had significant SVOO or arch anomalies, aortic crossclamp time, cardiopulmonary bypass time, preoperative AR, or preoperative PR. Postoperative deterioration in $\mathrm{AR}$, which ranged from none to trivial, occurred in 2 patients in the double-barrel DKS group and was more frequent in the double-barrel DKS group $(P=.048)$. Deterioration in PR was found in
4 patients in the end-to-side DKS group and in 1 patient in the double-barrel DKS group $(P=.001)$. The degree of $\mathrm{PR}$ deterioration ranged from none to mild in 1 patient, none to trivial in 2 patients, and trivial to mild in 2 patients. All instances of a deterioration of the semilunar valve regurgitations were observed at the first echocardiographic examination after the operation, and no further deterioration was observed in the late follow-up period. A multiple logistic regression analysis showed the double-barrel DKS to be superior to the end-to-side DKS for preventing the deterioration of postoperative PR (odds ratio 0.068; confidence interval, $0.007-0.688 ; P=.023$ ).

\section{DISCUSSION}

Although some reports have described the outcome of the pulmonary valve subjected to systemic circulation after an arterial switch operation or a Ross operation, few studies have attempted to describe the pulmonary valve function after the DKS operation. However, some reported the development of mild or moderate insufficiency of the pulmonary 

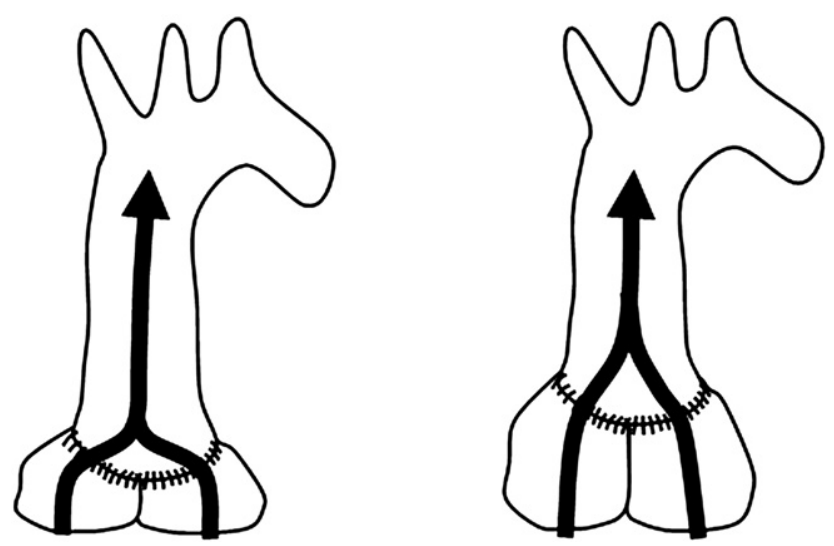

FIGURE 2. The effect of the length of the main pulmonary artery. The length of the main pulmonary artery is important to make a smooth systemic ventricular outflow tract.

valve function after the DKS operation. These reports mainly included patients undergoing the end-to-side DKS, and no reports have elucidated the pulmonary valve function after the double-barrel DKS. The incidence of deteriorated pulmonary valve function after the end-to-side DKS ranged from $4 \%$ to $55 \% .^{8-11}$ Thirty-one percent of the patients who underwent the end-to-side DKS in the current series had mildly deteriorated pulmonary valve insufficiency after the DKS operation. On the other hand, the incidence of deteriorated pulmonary valve insufficiency in patients with the double-barrel DKS was $2.9 \%$. The double-barrel DKS seems to be superior to the end-to-side DKS for pulmonary valve function. All instances of PR deterioration were observed during the early postoperative period. Therefore, the main cause of the impaired pulmonary valve function was thought to be a deformation of the pulmonary valve resulting from the DKS operation. The double-barrel DKS has been regarded as the first choice of treatment since 2002. The double-barrel DKS has a more smooth blood flow of the left ventricular outflow tract than the end-to-side DKS, thus inducing less energy loss and stress on the pulmonary valve, and there seems to be less potential for pulmonary valve deformation (Figure 1). Deterioration of the pulmonary valve function was more common in the patients with the endto-side DKS in this study than with the double-barrel DKS. Although this change in the pulmonary valve function was mild, the pulmonary valve function should be carefully followed because the long-term results are unknown. On the other hand, the deterioration of AR was more frequent in the double-barrel DKS group than in the end-to-side DKS group probably because the end-to-side DKS achieves a smoother blood flow of the ascending aorta and less tension of the aortic sinus than the double-barrel DKS (Figure 1). However, the degree of $\mathrm{AR}$ and $\mathrm{PR}$ deterioration was small and not thought to affect patients' circulatory conditions. There was no postoperative PR that was more than mild and no postoperative AR that was more than trivial. These results indicate the superiority of our careful consideration for the management strategy of semilunar valve functions in DKS candidates.

No patch materials were used for the DKS operation except for 1 patient who underwent the end-to-side DKS. The advantage of the double-barrel DKS is that a high-quality DKS anastomosis can be created to establish smooth systemic blood flow with no deformation of the pulmonary sinus without using patch materials. The double-barrel DKS is thought to be meaningful for the creation of the DKS anastomosis because homograft material is still not allowed for use in Japan. The length of the main pulmonary artery is important to create a smooth systemic ventricular outflow tract in the DKS operation (Figure 2). Therefore, PAB should be performed at a position as far from the pulmonary valve as possible to obtain a sufficient length of the main pulmonary artery for the DKS operation and avoid any deformation or degeneration of the pulmonary valve. The PAB is usually performed via a median sternotomy, not via a thoracotomy, if the patient is thought to require the DKS operation afterward because accurate positioning of the $\mathrm{PAB}$ is often more difficult via a thoracotomy than via a median sternotomy. The preservation of good pulmonary valve function and a sufficient length of the pulmonary trunk are important factors for achieving an excellent outcome when performing the DKS operation.

The double-barrel DKS cannot be performed in a patient who has a narrow ascending aorta. In this case, an end-toside DKS or a Norwood-type aortopulmonary anastomosis should be selected. The Norwood-type aortopulmonary anastomosis has been the first choice for such patients since 2002. Therefore, the end-to-side DKS has been performed only in patients with a long pulmonary trunk. It is important to avoid a deformation of the pulmonary valve when performing an end-to-side DKS in a patient with an insufficiently long pulmonary trunk. Patch material may be needed to maintain a smooth and optimal shape of pulmonary sinus for the end-to-side DKS (Figure 3).

If such patients have either pulmonary stenosis or PR that is deemed to be more than moderate, a DKS cannot be performed even if a patient requires it. However, in our experience, when patients have a pulmonary stenosis, they usually have a large bulboventricular foramen and subaortic space. Therefore, the number of patients who underwent SVOO release, except for DKS, in the Okayama University Hospital was small. One patient underwent a subaortic muscle resection before the Fontan operation. In addition, 4 patients underwent subaortic muscle resection or subaortic ridge resection at the Fontan operation. There were no Fontan candidates who underwent an enlargement of the bulboventricular foramen or a palliative arterial switch in the Okayama University Hospital.

Although there were 4 deaths in this study, no death was related to the DKS operation itself. On the other hand, 2 patients with SVOO died during the early postoperative period. 

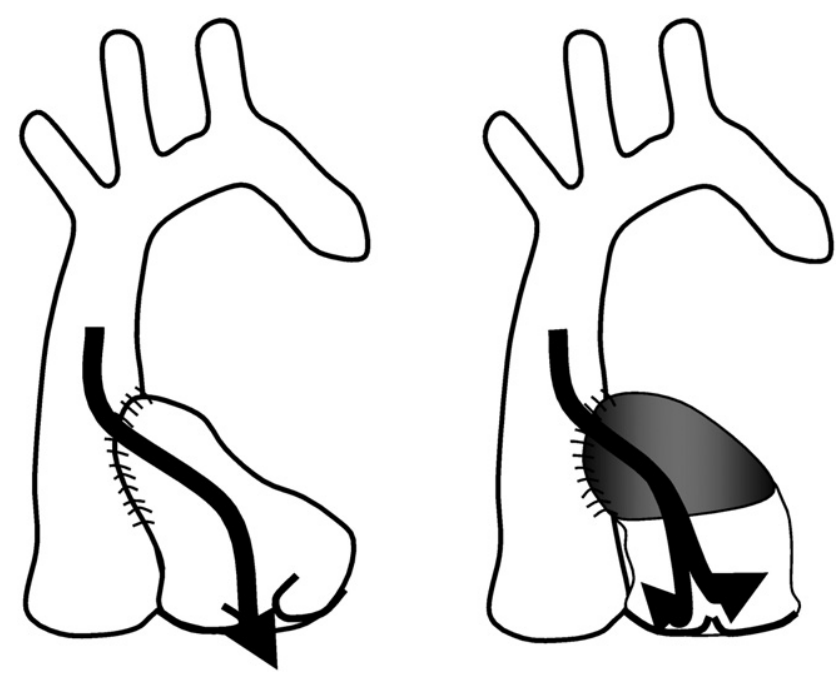

FIGURE 3. End-to-side DKS with a patch material. Some kind of a patch material may be needed to create a smooth and optimal shape for the end-toside DKS.

One early death was due to postoperative necrosis of the intestine. This patient had mild subaortic stenosis, heart failure, acidosis, and severe hypoxia preoperatively. Subaortic stenosis may have been underestimated because of the low output state; therefore, it was difficult to determine how the degree the subaortic stenosis contributed to the heart failure. This patient's condition did not improve, and acidosis persisted even after the operation. The preoperative SVOO might have contributed to the poor preoperative condition of this patient and thereby affected the results; therefore, the DKS should have been performed earlier in this case. Another early death occurred because of low output syndrome caused by SVOO immediately after a TCPC without a DKS operation. This patient could have survived if the DKS operation had been performed concomitantly with the TCPC initially. The DKS operation should be performed before or during the Fontan operation in patients with a potential for systemic ventricular outflow tract obstruction, such as tricuspid atresia with transposition of the great arteries and double-inlet left ventricle with transposition of the great arteries ${ }^{12}$ because SVOO is an independent risk factor for an early death after a Fontan operation and sometimes progresses significantly after a Fontan operation. ${ }^{13}$

\section{Study Limitations}

There are some limitations to this study. First, this study was conducted retrospectively and the selection of the DKS method was not randomized. Second, the number of patients was not significantly large and only 5 patients exhibited a deteriorated postoperative pulmonary valve function. Third, the preoperative and postoperative evaluations of pulmonary valve functions were performed using only echocardiography. The exact evaluation of pulmonary valve function is sometimes difficult and may depend on the skill of the echocardiographer. However, the echocardiographers (independent cardiac pediatrician) exchanged the patients' echocardiographic information with each other. They took special care to obtain consistent findings of echocardiography. In addition, all of the deteriorations of the pulmonary valve functions were observed during the first echocardiographic examination after the operation. In most of the cases, an echocardiographer checked the preoperative and early postoperative echocardiographic examinations. Therefore, there was little possibility of an incorrect evaluation of the pulmonary valve function. Finally, the deteriorations of the pulmonary valve functions in this study were considered to be minimal. Therefore, whether these deteriorated pulmonary valve functions might actually affect the long-term outcomes should be verified by further investigations. However, the fact that only a minimal deterioration of the pulmonary valve function was observed is important because it indicates the efficacy of the protocol for preserving the aortic and pulmonary sinuses. PR after a DKS operation should not become a problem even after long-term follow-up if the pulmonary valve is normal before the DKS operation.

\section{CONCLUSIONS}

The mid- to long-term clinical results of the DKS operation were excellent with a high rate of Fontan completion and low rate of postoperative deterioration of the pulmonary valve insufficiency. In addition, the double-barrel DKS is considered to be superior to the end-to-side DKS to preserve the postoperative pulmonary valve function when the DKS anastomosis is created without patch material. On the other hand, the double-barrel DKS may cause a deterioration of AR. Both cases of deteriorated semilunar valve regurgitations were thought to be due to the deformity of the pulmonary or aortic sinus caused by the DKS operation. Therefore, special care should be paid to prevent these deformities. The results of this study indicate that the pulmonary valve will be durable against the systemic circulation even in the longterm follow-up if the aortopulmonary anastomosis is appropriately created.

\section{References}

1. Damus PS. Correspondence. Ann Thorac Surg. 1975;20:724-5.

2. Kaye MP. Anatomic correction of transposition of great arteries. Mayo Clin Proc. 1975;50:638-40.

3. Stansel HC Jr. A new operation for d-loop transposition of the great vessels. Ann Thorac Surg. 1975;19:565-7.

4. Waldman JD, Lambertti JJ, George L, Kirkpatrick SE, Mathewson JW, Spicer RL, et al. Experience with Damus procedure. Circulation. 1988;78(5 Pt 2):III32-9.

5. Hiramatsu T, Imai Y, Kurosawa H, Takanashi Y, Aoki M, Shinoka T, et al. Midterm results of surgical treatment of systemic ventricular outflow obstruction in Fontan patients. Ann Thorac Surg. 2002;73:855-60.

6. Gates RN, Laks H, Elami A, Drinkwater DC Jr, Pearl JM, George BL, et al. Damus-Stansel-Kaye procedure: current indications and results. Ann Thorac Surg. 1993;56:111-9. 
7. Freedom RM, Williams WG, Fowler RS, Trusler GA, Rowe RD. Tricuspid atresia, transposition of the great arteries, and banded pulmonary artery. Repair by arterial switch, coronary artery reimplantation, and right atrioventricular valved conduit. J Thorac Cardiovasc Surg. 1980;80:621-8.

8. Huddleston CB, Canter CE, Spray TL. Damus-Kaye-Stansel with cavopulmonary connection for single ventricle and subaortic obstruction. Ann Thorac Surg. 1993; 55:339-45.

9. Di Donato RM, Amodeo A, Di Carlo DD, Galletti L, Rinelli G, Pasquini L, et al. Staged Fontan operation for complex cardiac anomalies with subaortic obstruction. J Thorac Cardiovasc Surg. 1993;105:398-404.
10. Odim JN, Laks H, Drinkwater DC Jr, George BL, Yun J, Salem M, et al. Staged surgical approach to neonates with aortic obstruction and single-ventricle physiology. Ann Thorac Surg. 1999;68:962-7.

11. Amin Z, Backer CL, Duffy CE, Mavroudis C. Does banding pulmonary artery affect pulmonary valve function after the Damus-Kaye-Stansel operation? Ann Thorac Surg. 1998;66:836-41.

12. Lan YT, Chang RK, Laks H. Outcome of patients with double-inlet left ventricle of tricuspid atresia with transposed great arteries. J Am Coll Cardiol. 2004;43:113-9.

13. Freedom RM. Subaortic obstruction and the Fontan operation. Ann Thorac Surg. 1998;66:649-52. 